\title{
Designing and testing the coronagraphic Modal Wavefront Sensor: a fast non-common path error sensor for high-contrast imaging
}

\author{
M.J. Wilby ${ }^{\mathrm{a}}$, C.U. Keller ${ }^{\mathrm{a}}$, S. Haffert ${ }^{\mathrm{a}}$, V. Korkiakoski ${ }^{\mathrm{b}}$, F. Snik ${ }^{\mathrm{a}}$, and A.G.M Pietrow ${ }^{\mathrm{a}}$ \\ ${ }^{a}$ Leiden Observatory, Leiden University, P.O. Box 9513, 2300 RA Leiden, The Netherlands \\ ${ }^{\mathrm{b}}$ Australian National University, Acton ACT 2601, Canberra, Australia
}

\begin{abstract}
Non-Common Path Errors (NCPEs) are the dominant factor limiting the performance of current astronomical high-contrast imaging instruments. If uncorrected, the resulting quasi-static speckle noise floor limits coronagraph performance to a raw contrast of typically $10^{-4}$, a value which does not improve with increasing integration time. The coronagraphic Modal Wavefront Sensor (cMWS) is a hybrid phase optic which uses holographic PSF copies to supply focal-plane wavefront sensing information directly from the science camera, whilst maintaining a bias-free coronagraphic PSF. This concept has already been successfully implemented on-sky at the William Herschel Telescope (WHT), La Palma, demonstrating both real-time wavefront sensing capability and successful extraction of slowly varying wavefront errors under a dominant and rapidly changing atmospheric speckle foreground. In this work we present an overview of the development of the cMWS and recent first light results obtained using the Leiden EXoplanet Instrument (LEXI), a high-contrast imager and high-dispersion spectrograph pathfinder instrument for the WHT.
\end{abstract}

Keywords: Adaptive Optics, Focal-Plane Wavefront Sensing, High-Contrast Imaging, Non-Common Path Errors, Holography

\section{INTRODUCTION}

Non-common path wavefront errors (NCPEs) are inherent in all systems using conventional pupil-plane wavefront sensors, which by necessity require there to be a difference in optical path between the wavefront sensor and science focal plane. While negligible for most science cases in astronomy, these minor aberrations represent a critical performance barrier for high-contrast imaging instruments including GPI at Gemini South, ${ }^{1}$ SPHERE at the $\mathrm{VLT}^{2}$ and ScExAO at Subaru, ${ }^{3}$ for which the resulting quasi-static speckle (QSS) background limits the raw $5 \sigma$ contrast achievable with coronagraphic high-contrast imaging techniques to approximately $10^{4} 10^{5}$, a value which does not improve with increasing integration time. ${ }^{4}$ The power spectrum and coherence timescales of these aberrations vary from instrument to instrument depending upon the specific thermal and vibrational environment. In general however, wavefront error amplitudes decrease and coherence timescales shorten with increasing spatial frequency of the aberration in the telescope pupil plane. This means that speckle suppression algorithms which fall into the two main families of angular differential imaging ${ }^{5-7}$ and spectral differential imaging ${ }^{8,9}$ are increasingly effective at wider radial separations, where the amount of field rotation and radial dispersion of speckles in a given image sequence is larger. This means NCPEs most severely limit the innerworking angle (IWA) of coronagraphs, although other factors such as image jitter due to system vibrations ${ }^{10}$ and the low-wind effect seen a SPHERE ${ }^{11}$ often limit current instrument performance in practice. Nonetheless, a stable real-time solution will be required for the next generation of instruments for ELT-class telescopes, such as the Exoplanet Imaging Camera and Spectrograph (EPICS ${ }^{12}$ for the E-ELT which aims to directly image rocky exoplanets at visible wavelengths, requiring contrast ratios of better than $10^{-9}$ at spatial separations of 100 milli-arcseconds.

Author correspondence information:

M.J. Wilby. E-mail: wilby@strw.leidenuniv.nl, Telephone: +31 (0)71 5278120 
It is therefore desirable to develop a real-time NCPE sensor which operates on second-to-minute timescales (in order to match typical coherence times), which is ideally completely common-path with the science focal plane. A low-order sensor correcting the first few tens of modes can in principle already eliminate a significant fraction of the most troublesome speckles and greatly improve raw coronagraph contrast and inner-working angle. The coronagraphic Modal Wavefront Sensor (cMWS ${ }^{13}$ provides a promising solution for exactly this problem, which uses the principle of the holographic modal wavefront sensor (HMWS) ${ }^{14,15}$ to create multiple secondary copies of the stellar point-spread function (PSF) in the science focal plane, which may be viewed as an extension to the astrometric and photometric spots used by GPI. ${ }^{16}$ By independently biasing these secondary copies with aberration modes drawn from a chosen basis set, the cMWS is able to retrieve an instantaneous modal decomposition of the low-order wavefront present at the science focal plane without interrupting observations. Furthermore, this technique is ideal for combination with the apodising phase plate coronagraph (APP) ${ }^{17-20}$ into a single phase element. The goal of this exercise is then to trade a small amount of throughput to the science PSF ( $\sim 1 \%$ per sensing mode), in order to optimally clean up to coronagraphic dark hole and thus gain multiple orders of magnitude in terms of raw contrast performance over the QSS-limited case.

The underlying principle of the cMWS has already been verified on-sky at the William Herschel Telescope (WHT), where is was shown to be able to accurately retrieve injected wavefront error during both narrowband and broadband operation. ${ }^{13}$ This paper therefore presents further work on the optimisation and preliminary closed-loop testing of the cMWS as part of the Leiden EXoplanet Instrument (LEXI) pathfinder for the WHT.

\section{THE CORONAGRAPHIC MODAL WAVEFRONT SENSOR (CMWS)}

This section provides an overview of the generation procedure and operation principles of the cMWS, including a summary of the most relevant equations. A full presentation of the sensor principle and first validation results can be found Wilby et al. 2016. ${ }^{13}$

Fig. 1 shows the principle behind creating a single-mode computer-generated hologram (CGH) $H_{k}$, which in this example is chosen to sense coma (Zernike mode $Z_{6}$ ). The bias wavefront corresponding to the aberration mode to be sensed is combined with a high-amplitude tilted reference wave which serves to spatially separate the secondary PSF copy from the zeroth order in the focal plane (panels 1 and 2 of Fig. 1 respectively). The resulting continuous phase hologram is spatially encoded by taking the cosine of the total phase screen (panel 3 ), which may be defined by analogy with the optical exposure process of physical holograms as

$$
\begin{aligned}
H_{k}(u, v) & =O_{k}^{*} R_{k}+O_{k} R_{k}^{*} \\
& =2 \cos \left(b_{k} M_{k}(u, v)+2 \pi\left(f_{k u} u+f_{k v} v\right)\right),
\end{aligned}
$$

where $O_{k}(u, v)=e^{i b_{k} M_{k}(u, v)}$ is the object (bias) wavefront for a RMS amplitude $b_{k}$ of the chosen bias mode $M_{k}(u, v)$ (in this case $Z_{6}(u, v)$ ), and $R_{k}(u, v)=e^{2 i \pi\left(f_{k u} u+f_{k v} v\right)}$ is the tilted reference wavefront with a total amplitude of $f_{k u, v}$ waves along each axis. In this way, the $H_{k}$ becomes effectively a modified diffraction grating, in which the diffraction orders $\geq \pm 1$ now contain the desired bias aberration. Note that throughout this section $(u, v)$ coordinate space denotes pupil-plane quantities and $(x, y)$ coordinates denote the focal-plane. The optimal amplitude of the bias wavefront is typically between 0.7 and 1.5 radians RMS, ${ }^{15}$ while the tilted reference wave is typically greater than 10 waves in amplitude in order to sufficiently separate the PSF copies from the zeroth order science PSF.

When illuminated with an aberrated wavefront $\Psi=A(u, v) e^{i \phi(u, v)}=A(u, v) e^{i \sum_{j} a_{j} M_{j}(u, v)}$ consisting of modal coefficients $a_{j}$ in the sensing basis (for a given telescope aperture function $A(u, v)$ ), the resultant focal-plane intensity pattern is given by $I=\left|\mathcal{F}\left[H_{k} \Psi\right]\right|^{2}$ (panel 4 of Fig. 1), where $\mathcal{F}$ is the Fourier Transform operator. This results in the creation of two conjugate PSF copies in the \pm 1 diffraction orders of the hologram, for which the on-axis intensity is described by

$$
I_{k \pm}(x, y)=\underbrace{\delta\left(\mathbf{x} \pm \mathbf{x}_{\mathbf{k}}\right)}_{\text {Carrier Frequency }} *|\mathcal{F} \underbrace{\left[e^{i\left(a_{k} \pm b_{k}\right) M_{k}(u, v)}\right.}_{\text {Wavefront Bias }} \underbrace{\left.e^{i \sum_{j \neq k} a_{j} M_{j}(u, v)}\right]}_{\text {Inter-Modal Crosstalk }}|^{2},
$$




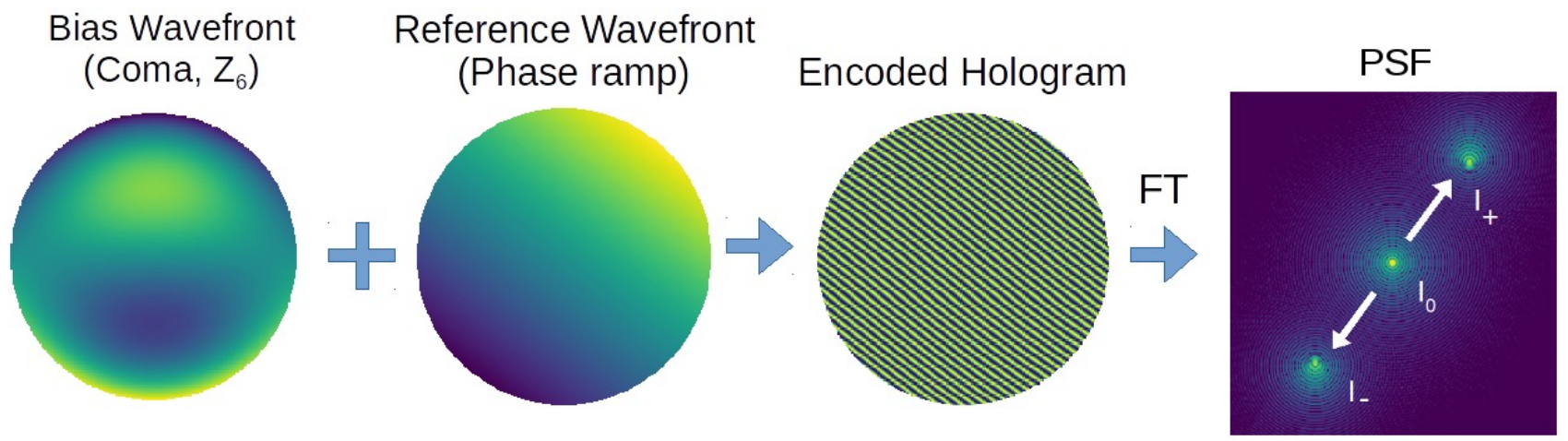

Figure 1. Creation of a single-mode computer-generated hologram $(\mathrm{CGH})$ sensitive to Coma $\left(Z_{6}\right)$. Panels 1-3: Phase maps of the bias wavefront $M_{k}(u, v)=Z_{6}\left(b_{k}=0.7\right.$ radians RMS), tilted reference wavefront (10 waves amplitude) and encoded hologram phase map from Eq. 2 respectively. Panel 4: Corresponding simulated PSF showing \pm 1 diffraction orders containing opposite wavefront bias, positioned symmetrically about the zeroth order unbiased PSF. Note: Throughout this paper the "viridis" python colour map (green/blue) refers to simulations while the "magma" colour map (orange/purple) presents laboratory or on-sky images.

Multi-Mode Hologram
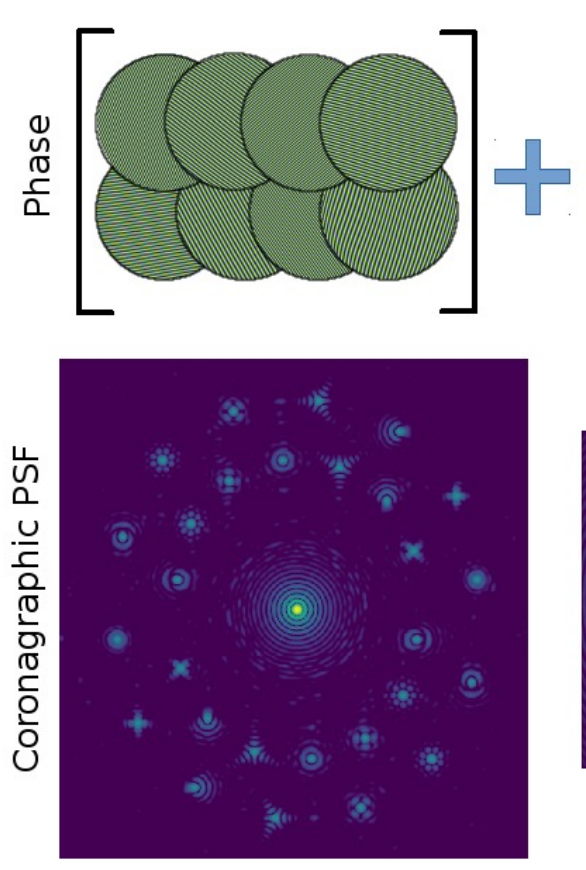

APP Coronagraph
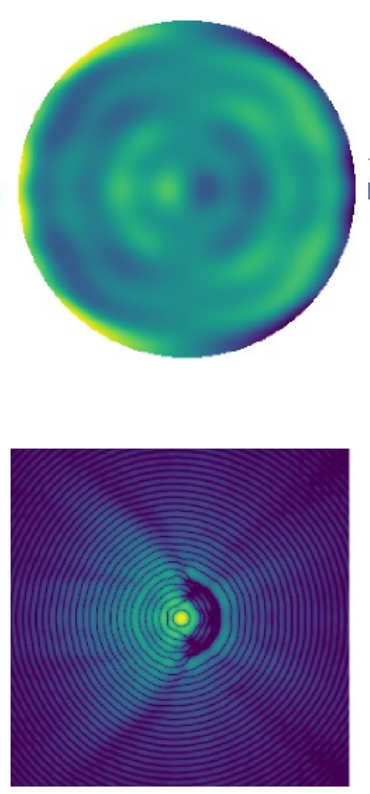
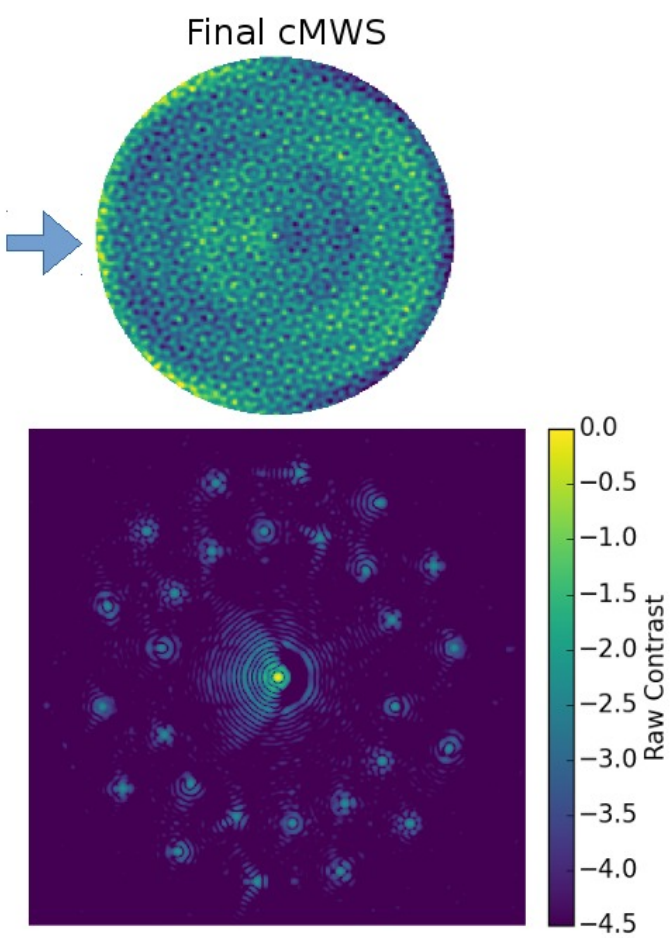

Figure 2. Diagram illustrating the multiplexing procedure of a cMWS sensitive to 14 low-order Zernike modes, with an apodising phase plate (APP) coronagraph optimised to create a $180^{\circ}$ dark hole spanning radial separations from $2.5-6 \lambda / D$ capable of reaching $10^{-6}$ in contrast.

where $\delta\left(\mathbf{x} \pm \mathbf{x}_{\mathbf{k}}\right)$ determines the relative position of PSF copies in the focal plane, $\mathcal{F}$ is the Fourier transform operator and $*$ is the convolution operator. Crucially, these wavefront sensing spots contain opposite signs of the wavefront biases such that the difference term $\left(a_{k} \pm b_{k}\right) M_{k}(u, v)$ leads to an asymmetry in Strehl ratio between the \pm 1 diffraction orders when a non-zero coefficient of the aberration mode $a_{k}$ is present in the system. The final term represents the convolution of each PSF with all remaining modes present in the aberrating wavefront.

This principle is then easily extended to multi-mode holograms by summation of multiple CGHs containing different bias modes and tilted reference waves. The \pm 1 orders created by each hologram are functionally independent of each other provided they are sufficiently separated in the focal plane, and as the zeroth order 

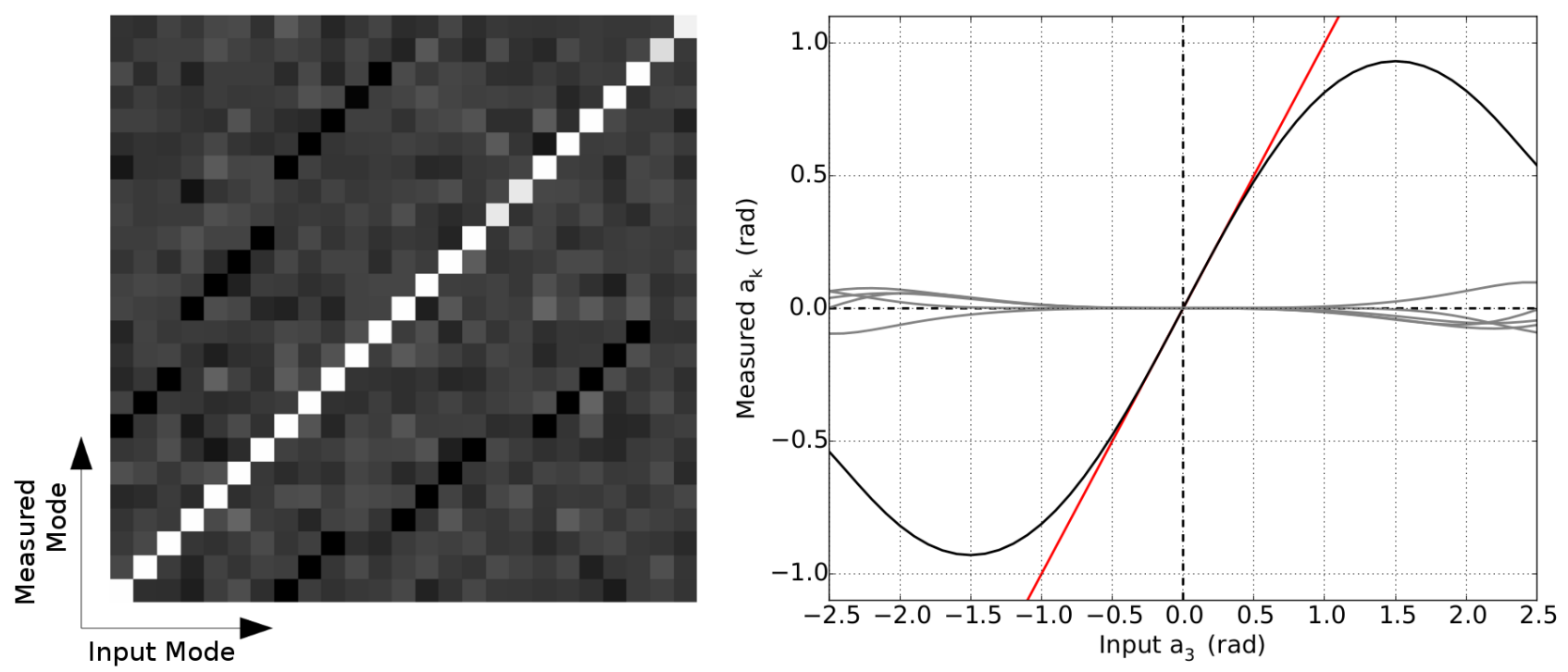

Figure 3. Left: Interaction matrix of a 25-mode Zernike cMWS formed from the gradients in sensor response (Eq. 4) as a function of injected wavefront error, which can be seen to be highly diagonal. Right: Simulated response curve of focus mode $\left(Z_{3}\right.$, black) and inter-modal crosstalk with other first 5 modes (grey) after calibration with the aforementioned interaction matrix. The 1:1 correspondence line is shown in red, which is consistent with modal response for $a_{3}<$ 0.5 radians.

is effectively a leakage term of the hologram it sees none of the bias aberration, allowing it to be used for coronagraphic observations. Fig. 2 illustrates this final multiplexing process which combines multiple singlemode holograms corresponding to the 14 lowest-order non-trivial Zernike modes with an apodising phase plate coronagraph (APP) to create the final cMWS optic, capable of performing simultaneous wavefront sensing and high-contrast imaging observations using the zeroth order PSF. It should be noted that this technique is very similar in principle to that of the vector apodising phase plate (vAPP) coronagraph, ${ }^{19,20}$ which uses circular polarisation splitting to create two bright spatially separated conjugate PSFs capable of simultaneously probing a full $360^{\circ}$ search region. The objective of the cMWS is however to create many low-amplitude PSF copies for wavefront sensing, while maximising the "leakage term" intensity for science observations. These two techniques are entirely compatible and a prototype vector-cMWS has already been manufactured on a liquid crystal phase plate using direct-writing techniques. ${ }^{21}$

The relative Strehl ratios of wavefront sensing spots created in the focal-plane must now be measured and calibrated so as to return the modal decompositions coefficients of $\phi=\sum_{k} a_{k} M_{k}(u, v)$ with minimal computational overhead. In practice this is achieved using normalised intensity measurements $I_{k}=\left(I_{k+}-I_{k-}\right) /\left(I_{k+}+I_{k-}\right)$ of the respective Airy cores of each PSF copy to ensure independence of fluctuations in total intensity, while integration of the full Airy core $(r=1.22 \lambda / D)$ provides improved signal-to-noise ratio and stability against small tip-tilt misalignments with respect to the extraction region of interest used for wavefront sensing. From the intensity measurements of the Airy core of each sensing spot from Eq. 3, it is then necessary to construct the associated interaction matrix $\hat{\mathbf{S}}$ formed from the gradients of measured modal response curves as a function of known input aberration

$$
S_{i j}=\left.\frac{\delta I_{i}}{\delta a_{j}}\right|_{I=0},
$$

Fig. 3a shows the response matrix formed from these gradients which can be seen to be highly diagonal, with a mean on-diagonal components of $1.03 \pm 0.01$ compared to mean off-diagonal response of $-0.0169 \pm 0.0001$, however, some residual anti-correlations can be seen due to minor sources of inter-modal crosstalk. The final calibration of measured wavefront is then achieved using the inverted matrix

$$
\mathbf{a}=\hat{\mathbf{S}}^{-1} \mathbf{I}-\mathbf{a}_{\mathbf{0}}
$$



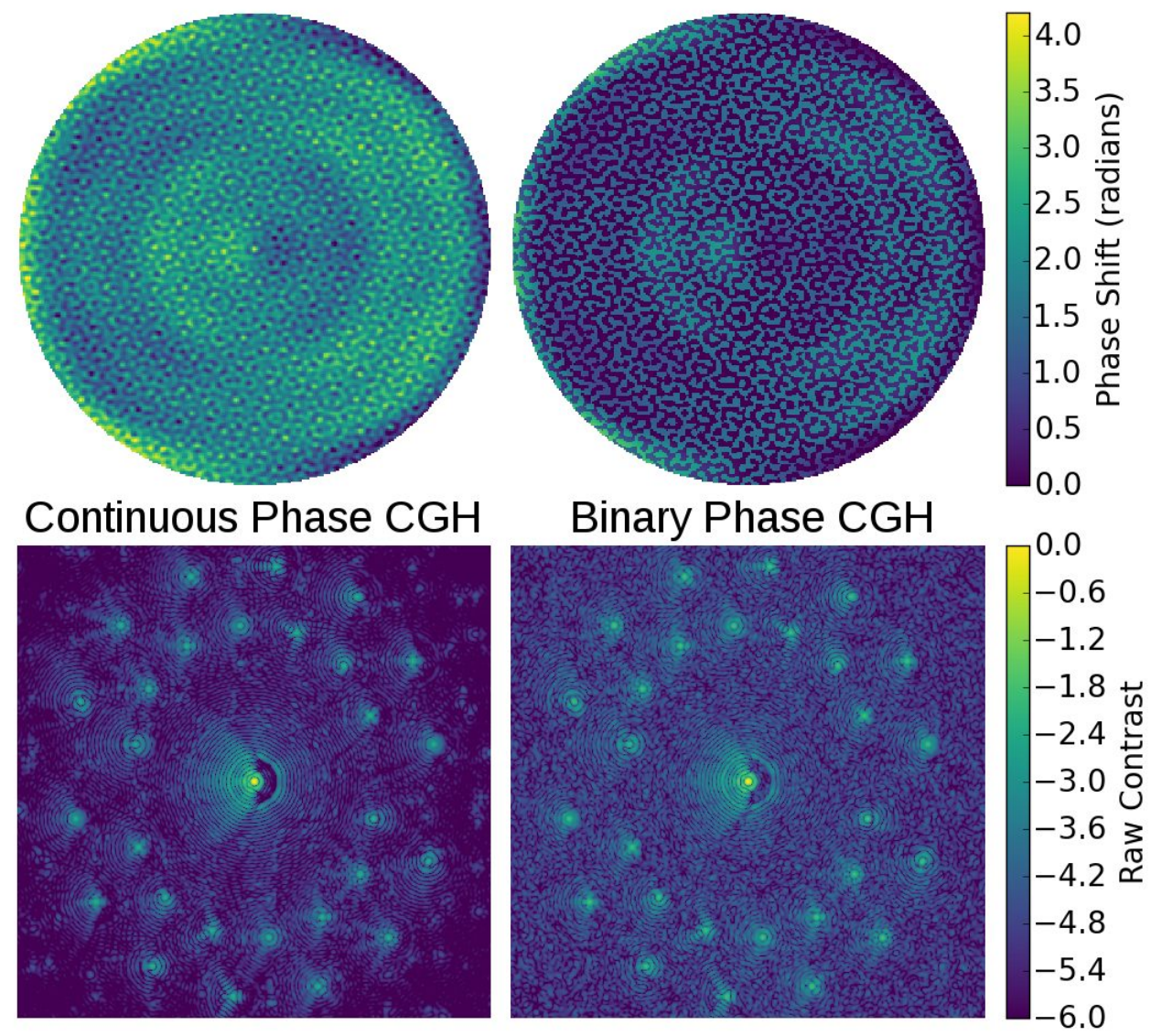

Figure 4. Left: Continuous phase hologram pupil phase phase map (top) and focal-plane PSF (bottom), for the 14 Zernike modes, $180^{\circ}$ dark hole cMWS design of Fig. 2. Right: Equivalent binary-hologram cMWS with PSF copies of the same $10^{-3}$ contrast with respect to the science PSF. Note the decreased stroke required to implement the binary hologram (with a CGH amplitude of $0.5 \pi$ as opposed to $1.3 \pi$ radians), at the cost of creating a $10^{-5}$ contrast speckle background.

where $\mathbf{a}$ is the final vector of wavefront coefficients (in units of radians RMS), $\hat{\mathbf{S}}^{-\mathbf{1}}$ is the inverted interaction matrix and $\mathbf{I}$ is the vector of normalised intensity measurements. $\mathbf{a}_{\mathbf{0}}$ is an additional vector of reference offsets corresponding to the measured modal coefficients of the APP, which is necessary in order to prevent the coronagraph phase pattern from being partially erased during closed-loop correction. By performing this interaction matrix calibration the sensor is also able to provide a linear correction for inter-modal crosstalk and increase the effective dynamic range of the sensor. The corrected response curves of the six lowest order modes of the example cMWS is shown in Fig. $3 \mathrm{~b}$ as a function of introduced focus error $\left(Z_{3}\right)$, using simulated wavefront extraction and calibration with the interaction matrix of Fig. 3a. This shows that the sensor possesses a linear sensing regime within applied wavefront bias value $b_{k}= \pm 0.7$ radians, and has a turning point at $a_{3}=2 b_{k}$ after which the input aberration begins to dominate and sensor response correspondingly decreases. The other sensing modes, which have no component present in the aberrating wavefront $\Psi$, are well-constrained about zero across the majority of this range although some minor response can be seen at high input aberrations as non-linear crosstalk terms begin to become significant.

A practical consideration which must be considered when implementing the cMWS is whether to use a continuous or binary phase hologram. The latter has the advantage of significantly reducing the required phase stroke required to obtain a given diffraction efficiency and improves control over the relative brightness of PSF copies, but comes at the cost of creating a faint speckle background. All simulated cMWS designs presented up to this point have used the full continuous phase of Eq. 2, but a convenient binarising operation is to the argument of the multiplexed continuous phase hologram via 


$$
\left.\phi_{h}(x, y)=\frac{s}{\pi} \arg \left[\sum_{k}^{N} H_{k}(x, y)\right)\right],
$$

where $s$ is the desired binary hologram amplitude in radians, which may be freely varied between 0 and $\pi$ to provide direct control over the total diffraction efficiency, where $s=\pi$ results in maximum intensity of wavefront sensing PSF copies and no power in the zeroth order PSF. By contrast, the continuous phase hologram has a highly non-linear relation between hologram amplitude and diffraction efficiency, ${ }^{22}$ which for the highest efficiencies also increasingly couples light into second order and cross-term PSF copies located elsewhere in the focal plane, thus reducing the Strehl ratio and increasing the risk of contamination-based inter-modal crosstalk. The performance of these two hologram designs is illustrated in Fig. 4, where it can be seen that the binarisation operation comes at the cost of a speckle background at a raw contrast of approximately $10^{-5}$, due to partial loss of information and thus fine phase control. The residual background structure in the continuous phase hologram is due to aforementioned second-order and cross-terms in the hologram components, however these are significantly more sparse than the speckle floor generated by the binary hologram; contamination may therefore be avoided by positioning PSF copies in a non-redundant pattern. The binary hologram is therefore preferable for practical implementation of the cMWS on a high-resolution spatial light modulator of limited stroke as is presented in this paper (see Sec. 3), however in terms of final commissioned designs manufactured using liquid crystal phase plates, it is preferable to use the continuous phase hologram in order to maximise the final limiting APP contrast.

\section{THE LEIDEN EXOPLANET INSTRUMENT (LEXI)}

LEXI is a pathfinder high-contrast instrument for the William Herschel Telescope (WHT), which is designed to combine the advantages of conventional coronagraphic high-contrast imaging with the template-based crosscorrelation techniques available to high-dispersion spectroscopy, ${ }^{23}$ in order to reach final contrasts of $10^{-6}$ $10^{-7} .^{24}$ Fig. 5 shows an overview of LEXI components based on an image of the assembled instrument at the WHT GHRIL Nasmyth platform during first light observations in June 2016, illustrating the three main arms of the instrument:

- Adaptive Optics: Consists of a ALPAO DM97-15 97-actuator deformable mirror driven by a 10x10 Shack-Hartmann wavefront sensor using an Andor Ixon DU879 EM-CCD, capable of delivering modest Strehl ratios of $\sim 15 \%$ at visible wavelengths $(630 \mathrm{~nm})$.

- High-Contrast Imager: A BNS-p512 liquid crystal spatial light modulator (LC-SLM) is used to implement cMWS phase patterns. A $512 \times 512$ pixel Andor Ixon+ 860 EM-CCD with a 4.7 " field of view is used to re-image the AO-corrected coronagraphic PSF and provide wavefront sensing information.

- High-Dispersion Spectrograph: A single long-slit spectrograph with a resolution of $\mathrm{R}=75,000$ across a $10 \mathrm{~nm}$ bandpass centered on $640 \mathrm{~nm}$, using an Andor Zyla 5.5 sCMOS camera. The spectrograph arm is designed to be common-path with the LC-SLM, in order to provide spectoscopy inside the coronagraphic dark hole.

Following initial on-sky verification carried out in April 2015 without access to adaptive optics, ${ }^{13}$ this instrument provides the logical next step for further on-sky testing of the cMWS by providing a high-resolution, AO-corrected imaging system which incorporates a spatial light modulator for flexible implementation of the necessary high-resolution phase designs. This allows for further testing of the cMWS for offline PSF optimisation and initial on-sky closed-loop testing; Fig. 6 shows the results of using a 20 Zernike mode cMWS for correction of static wavefront aberrations on the LEXI test bench in Leiden. This shows a gain in Strehl of $20 \%$ in 8 iterations, with final correction quality limited in practice by the maximum stroke of the BNS p512 SLM of 5.5 radians. This limitation prevents the control of arbitrarily large aberrations via phase wrapping (which is otherwise suitable for use with a high-resolution SLM) and thus cannot fully compensate regions of the pupil with the largest phase error. This will be solved in a scheduled upgrade to LEXI in which NCPE correction will be applied directly using the AO deformable mirror, allowing significant improvements in both maximum stroke and correction cadence. 


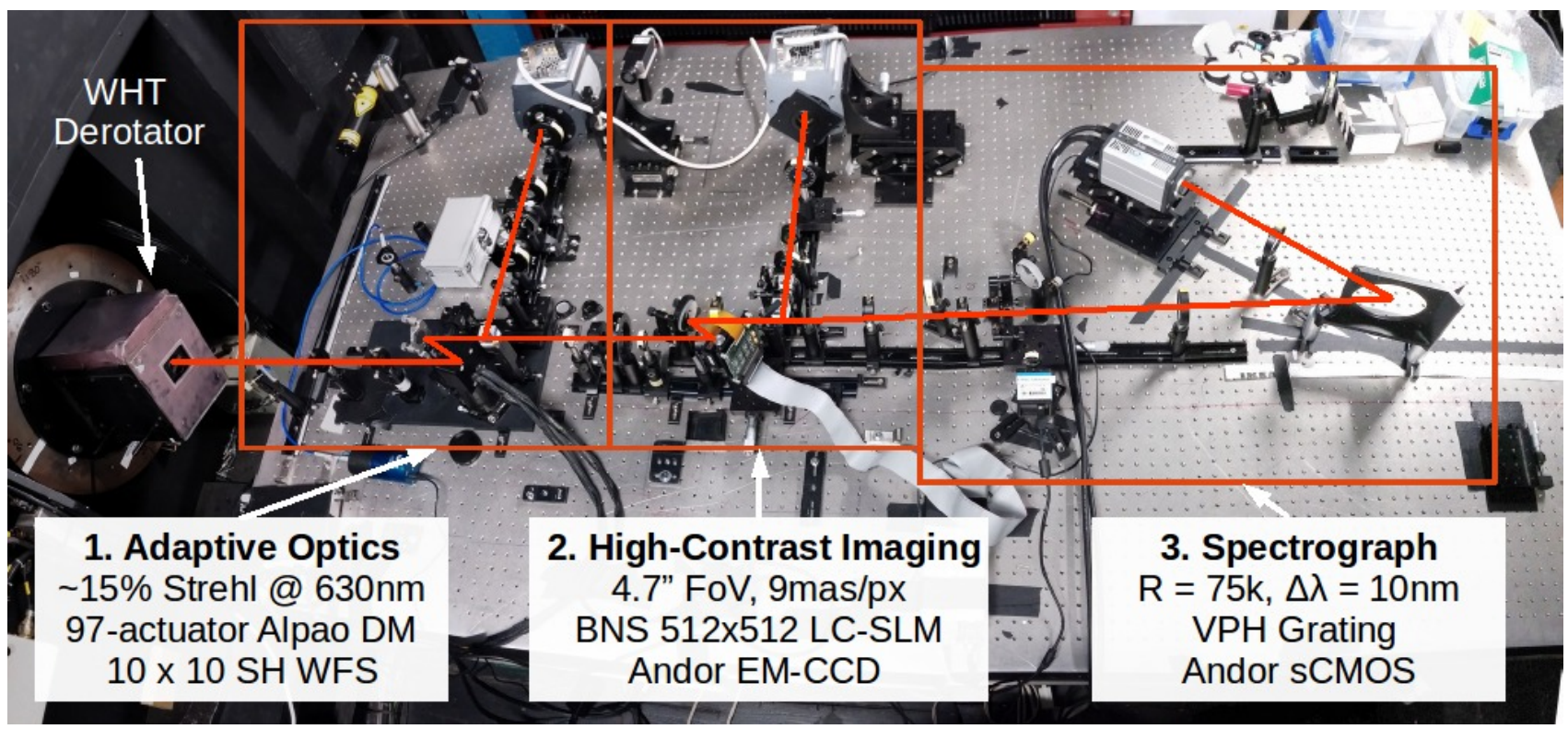

Figure 5. Image of LEXI on the WHT Nasmyth optical bench, with boxes and labels indicating the three main arms of the instrument. The optical path from the WHT derotator to each science camera is shown in red.


Figure 6. Closed-loop optimisation of the LEXI PSF using 20 Zernike mode sensing basis. Shown here is the central science PSF before correction (middle panel) and after 8 closed-loop iterations (right panel). The left panel shows image Strehl ratio (calculated by normalising images with respect to the $10 \lambda / D$ encircled energy of the simulated perfect PSF) as a function of closed-loop iteration number. Final correction quality is limited by the corrective stroke of the SLM; after 8 iterations this becomes saturated and no further significant gains in Strehl ratio are made. 


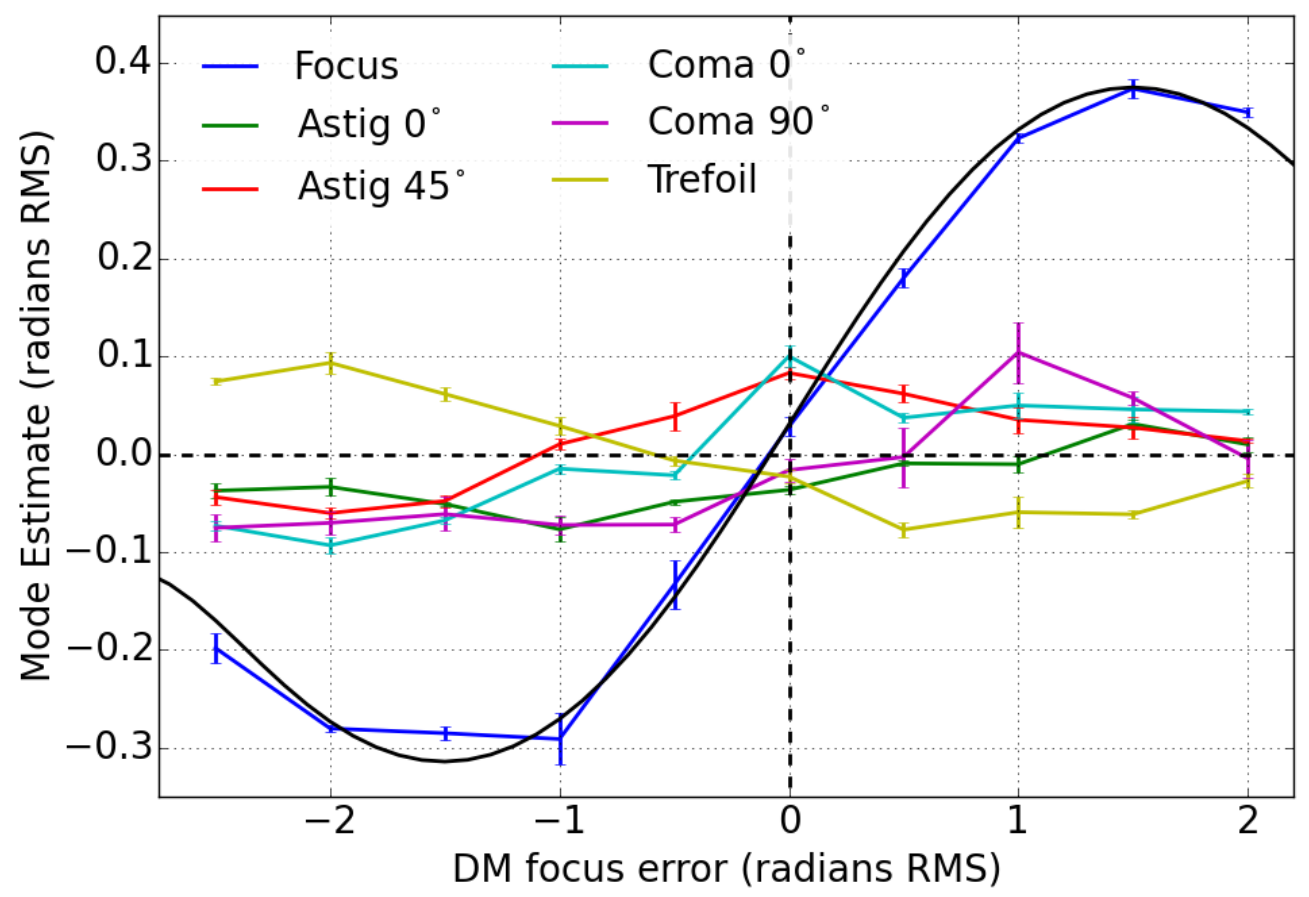

Figure 7. On-sky response of focus mode measured at 10s cadence, with known aberrations introduced via SH-WFS reference offsets on the deformable mirror. A scaled fit of the simulated curve of Fig. 3 is overlaid in black, highlighting good agreement between simulation and obtained response behaviour, albeit at reduced sensitivity.

\section{PRELIMINARY ON-SKY VALIDATION}

This section presents some of the preliminary results from the June 2016 observing trip, intended to validate the real-time sensing and closed-loop correction capabilities of the cMWS.

Fig. 7 shows the results of real-time on-sky passive wavefront sensing, intended to demonstrate the retrieval of known wavefront error systematically injected on the deformable mirror by applying reference offsets to the ShackHartmann wavefront sensor (SH-WFS). Wavefront estimates were obtained by centroiding extraction windows of radius $1.22 \lambda / D$ on each PSF copy to extract airy core intensities, which are then calibrated using the normalised intensity metric and simulated interaction matrix described in Sec. 2, as an experimentally-deduced interaction matrix was not available. Each data point represents the average of three 10s integrations while observing Vega, with the low cadence designed to fully average out incoherent atmospheric speckles, thus creating a smooth PSF which is consistent from frame-to-frame, allowing reliable sensing of the underlying NCPEs. Data points were not sampled in the order in which the x-axis of Fig. 7 is plotted, but in a pseudo-random order of oscillating sign in order to eliminate the possibility of changing seeing conditions or real changes in NCPEs from producing false correlations in the response plot. The simulated response curve of Fig. 3 is over-plotted in black, based on a 4-parameter fit, including $\mathrm{x}$ and $\mathrm{y}$ scaling and offsets from the origin to allow for unknown static aberrations and sensitivity variations; this can be seen to match well with the measured focus data (blue).

It can be seen that the response of non-focus modes is significantly elevated with respect to the minimal crosstalk behaviour of Fig. 3, with some notable correlated response most notably an anti-correlation with trefoil (yellow). This is not due to chance correlated changes in observing conditions as ensured by the pseudo-random order in which data was sampled, and from the consistency of data points with simulations across the entire response curve it can be inferred that such factors were not significant during the course of these observations. It is therefore likely that this is the result of inaccuracies in the theoretical response matrix calibration: any mis-match between the sensing basis and the modes actually implemented on the deformable mirror will result in similarly elevated crosstalk behaviour. In this scenario the presented response curves would reflect accurate 
sensor response to injected aberrations which are not purely defocus, however this cannot be verified using the available data and requires further investigation.

It must also be noted that the peak response amplitude of \pm 0.35 radians RMS obtained during real-time on-sky measurements is significantly lower than the \pm 0.95 radians response obtained in the simulated curve of Fig. 3. This simulated value was however also replicated during initial on-sky verification in April 2015, using deep stacks of nearly diffraction-limited images obtained by using only a $0.4 \mathrm{~m}$ WHT sub-aperture in the absence of $\mathrm{AO}$ correction, in order to limit the strength of atmospheric aberrations with respect to the diffraction limit. ${ }^{13}$ The cause of the observed reduction in sensitivity in this instance is not fully understood, however it is possibly due to the significantly increased relative aberration strength, with the AO system delivering approximately $15 \%$ image Strehl ratios under 0.8" seeing conditions. As the cMWS is optimised to operate with the presence of an Airy core, a predominately seeing-limited PSF will most likely lead to reduced sensor response with respect to an equivalent XAO system. It is however also possible for incorrect bias/dark subtraction or flat-fielding effects to bias the normalised intensity metric to lower values, which may contribute to reducing sensor response.

Datasets were also taken while operating the cMWS in closed-loop SLM correction mode, which remain to be analysed. It was promising to note however that the median PSF quality did not substantially diverge during this procedure, indicating at least non-destructive operation of the sensor for a total of 8 minutes at a 10s correction cadence under 0.7" seeing conditions. This and the outstanding questions regarding real-time sensor response will be the subject of future study once LEXI has been returned from the WHT to Leiden, as part of a scheduled instrument upgrade.

\section{CONCLUSIONS}

Current and future generations of high-contrast imaging instruments require a stable, real-time solution to NCPEs sensing and correction. The coronagraphic Modal Wavefront Sensor (cMWS) is a fast, truly common-path and broadband-compatible sensor which is designed to clean up the search regions of the apodising phase plate (APP) coronagraph. This may be optimised either as a low-order Zernike basis sensor to compensate the most troublesome low spatial frequency speckles which limit coronagraph inner working angles, or to use a custom sensing basis to efficiently correct residual intensity structure in a small APP dark hole, optimised for characterisation of known exoplanets. The cMWS has undergone preliminary validation in closed-loop simulations, the laboratory environment and at the William Herschel telescope with the Leiden EXoplanet Instrument (LEXI), in which it has been shown to be able to accurately recover known injected wavefront error during both narrowband and broadband passive sensing, in addition to performing preliminary closed-loop tests.

Future applications of the cMWS include wavelength-resolved wavefront sensing, to which the cMWS is naturally suited due to wavelength-dependent radial dispersion of the biased PSF copies, and fast $(>10 \mathrm{kHz})$ sensing and control of a limited number of sensing modes to take advantage of the minimal computational requirements of the cMWS. Electric field conjugation (EFC) is also being explored as a further extension of the holographic approach to focal-plane sensing, which uses two pairs of holographic copies containing known phase probes to linearly reconstruct the full electric field in the dark hole. This approach has been shown in simulations to reach $10^{-10}$ contrast with specially optimised APP coronagraphs for ELT-class telescopes, for sufficiently low initial wavefront error to be within the algorithm's linear correction regime. ${ }^{25}$

Immediate work will focus on a scheduled upgrade to the LEXI instrument as a high-contrast imaging test bed, which will provide a significant increase in NCPE correction performance. Further closed-loop validation may then be performed and the cMWS further optimised for applications including XAO systems for upgraded VLT or future ELT-class instruments, such as METIS and EPICS for the E-ELT.

\section{ACKNOWLEDGMENTS}

This work is funded by the Nederlandse Onderzoekschool Voor Astronomie (NOVA). The authors would also like to thank Emiel Por, David Doelman, Jens Hoeijmakers \& Steven Bos, as well as the WHT operations team, for their valuable assistance during LEXI first light observations. 


\section{REFERENCES}

[1] Macintosh, B., Graham, J. R., Ingraham, P., et al., "First light of the Gemini Planet Imager," Proceedings of the National Academy of Science 111, 12661-12666 (Sept. 2014).

[2] Beuzit, J.-L., Feldt, M., Dohlen, K., et al., "SPHERE: a 'Planet Finder' instrument for the VLT," in [Ground-based and Airborne Instrumentation for Astronomy II], Proc. SPIE 7014, 701418 (July 2008).

[3] Jovanovic, N., Martinache, F., Guyon, O., et al., "The Subaru Coronagraphic Extreme Adaptive Optics System: Enabling High-Contrast Imaging on Solar-System Scales," Publications of the Astronomical Society of the Pacific 127, 890-910 (Oct. 2015).

[4] Martinez, P., Loose, C., Aller Carpentier, E., and Kasper, M., "Speckle temporal stability in XAO coronagraphic images," A\&B 541, A136 (May 2012).

[5] Marois, C., Lafrenière, D., Doyon, R., Macintosh, B., and Nadeau, D., "Angular Differential Imaging: A Powerful High-Contrast Imaging Technique," The Astrophysical Journal 641, 556-564 (Apr. 2006).

[6] Lafrenire, D., Marois, C., Doyon, R., Nadeau, D., and Artigau, E., "A new algorithm for point-spread function subtraction in high-contrast imaging: A demonstration with angular differential imaging," The Astrophysical Journal 660(1), 770 (2007).

[7] Soummer, R., Pueyo, L., and Larkin, J., "Detection and Characterization of Exoplanets and Disks Using Projections on Karhunen-Love Eigenimages," The Astrophysical Journal Letters 755(2), L28 (2012).

[8] Marois, C., Phillion, D. W., and Macintosh, B., "Exoplanet detection with simultaneous spectral differential imaging: effects of out-of-pupil-plane optical aberrations," in [Society of Photo-Optical Instrumentation Engineers (SPIE) Conference Series], Proc. SPIE 6269, 62693M (June 2006).

[9] Rameau, J., Chauvin, G., Lagrange, A.-M., Maire, A.-L., Boccaletti, A., and Bonnefoy, M., "Detection limits with spectral differential imaging data," $A \mathscr{E} A \mathbf{5 8 1}$, A80 (Sept. 2015).

[10] Hartung, M., Hayward, T., Saddlemyer, L., et al., "On-sky vibration environment for the Gemini Planet Imager and mitigation effort," in [Adaptive Optics Systems IV], Proc. SPIE 9148, 91480N (Aug. 2014).

[11] Wilby, M., Keller, C., Sauvage, J., et al., "A "Fast and Furious" Solution to the Low-Wind Effect for SPHERE at the VLT," in [Adaptive Optics Systems V], Proc. SPIE 9909 (July 2016).

[12] Kasper, M., Beuzit, J.-L., Verinaud, C., et al., "EPICS: direct imaging of exoplanets with the E-ELT," Proc. SPIE 7735, 77352E-77352E-9 (2010).

[13] Wilby, M., Keller, C., Snik, F., Korkiakoski, V., and Pietrow, A., "The coronagraphic Modal Wavefront Sensor: a hybrid focal-plane wavefront sensor for high-contrast imaging," A\&A, submitted (2016).

[14] Neil, M. A. A., Booth, M. J., and Wilson, T., "New modal wave-front sensor: a theoretical analysis," $J$. Opt. Soc. Am. A 17, 1098-1107 (Jun 2000).

[15] Booth, M. J., "Direct measurement of Zernike aberration modes with a modal wavefront sensor," Proc. SPIE 5162, 79-90 (2003).

[16] Wang, J. J., Rajan, A., Graham, J. R., et al., "Gemini planet imager observational calibrations VIII: characterization and role of satellite spots," in [Ground-based and Airborne Instrumentation for Astronomy V], Proc. SPIE 9147, 914755 (July 2014).

[17] Codona, J. L., Kenworthy, M. A., Hinz, P. M., Angel, J. R. P., and Woolf, N. J., "A high-contrast coronagraph for the mmt using phase apodization: design and observations at 5 microns and $2 \lambda$ /d radius," Proc. SPIE 6269, 62691N-62691N-10 (2006).

[18] Kenworthy, M. A., Quanz, S. P., Meyer, M. R., et al., "An apodizing phase plate coronagraph for VLT/NACO," in [Ground-based and Airborne Instrumentation for Astronomy III], Proc. SPIE 7735, 773532 (July 2010).

[19] Snik, F., Otten, G., Kenworthy, M., et al., "The vector-APP: a broadband apodizing phase plate that yields complementary PSFs," in [Modern Technologies in Space- and Ground-based Telescopes and Instrumentation II], Proc. SPIE 8450, 84500M (Sept. 2012).

[20] Otten, G. P. P. L., Snik, F., Kenworthy, M. A., Miskiewicz, M. N., and Escuti, M. J., "Performance characterization of a broadband vector apodizing phase plate coronagraph," Opt. Express 22, 30287-30314 (Dec 2014).

[21] Miskiewicz, M. N. and Escuti, M. J., "Direct-writing of complex liquid crystal patterns," Opt. Express 22, 12691-12706 (May 2014). 
[22] Changhai, L., Fengjie, X., Shengyang, H., and Zongfu, J., "Performance analysis of multiplexed phase computer-generated hologram for modal wavefront sensing," Appl. Opt. 50, 1631-1639 (Apr 2011).

[23] Snellen, I., de Kok, R., Birkby, J. L., et al., "Combining high-dispersion spectroscopy with high contrast imaging: Probing rocky planets around our nearest neighbors," A\&\&A 576, A59 (Apr. 2015).

[24] Haffert, S., Keller, C. U., and Snellen, I. A. G., "The Leiden EXoplanet Instrument (LEXI): a high-contrast high-dispersion spectrograph," in [Ground-based and Airborne Instrumentation for Astronomy VI], Proc. SPIE 9908 (July 2016).

[25] Por, E. and Keller, C. U., "Focal-plane electric field sensing with pupil-plane holograms," in [Adaptive Optics Systems V], Proc. SPIE 9909 (July 2016). 